\title{
A LDPC-Coded Distributed Alamouti Space-time Relay Cooperation Scheme over Rayleigh Fading Channels
}

\author{
Lei Tang, Wei-Wei Liu \\ School of Communication Engineering, Nanjing Institute \\ of Technology, Nanjing, China \\ E-mail: tanglei811019@163.com
}

\author{
Tian-Yu Wang \\ FN Product MKT and Solution Team, ZTE Corporation, \\ Nanjing, China \\ E-mail: wang.tianyu@zte.com.cn
}

\begin{abstract}
An efficient distributed Alamouti Space-time coded relay cooperation scheme is proposed over a Rayleigh fading channel, where structured irregular systematic low-densityparity-check (LDPC) codes are employed. In the known channel state information (CSI) scenario, a concatenation of combiner and joint iterative decoder is effectively carried out in the destination. The theoretical analysis and numerical simulations show that the proposed LDPC-coded cooperation scheme can well combine diversity and channel coding gain, and clearly outperforms the conventional cooperation under the same conditions.
\end{abstract}

Keywords-alamouti space-time block coding; coded relay cooperation; LDPC codes; joint iterative decoding

\section{INTRODUCTION}

Cooperative communication [1] is an effective technique to achieve large capacity and to combat fading environment. The basic idea of cooperative communications is that the mobile users equipped with single antenna share their antennas and form virtual antenna arrays to emulate multiple-input multiple-output (MIMO) system. Recent studies have proposed three main categories of protocols that support cooperative communications, i.e., amplify-andforward (AF) [2] detect-and-forward (DF) [3] and coded cooperation protocols (CC) [4-8]. In order to achieve better diversity, distributed space-time block codes (STBCs) have been proposed for $\mathrm{AF}$ and $\mathrm{DF}$ protocols in the literature [911]. Motivated by these results, in this paper, we introduce a kind of distributed Alamouti space-time relay cooperation scheme based on CC protocols. Compared with $\mathrm{AF}$ and $\mathrm{DF}$ protocols, CC protocol has higher transmission reliability. Furthermore, since the Alamouti STBC can obtain not the coding gain but the diversity gain, the structured irregular systematic LDPC codes are employed in the proposed scheme to improve the error rate performance.

\section{SYSTEM DESCRIPTION}

The model of our proposed LDPC-coded distributed Alamouti space-time relay cooperation is depicted in Fig.1.

Firstly, the source (S) transmits codeword ${ }^{c_{1}}$ achieved by LDPC-1 to the relay (R). Secondly, R decodes the incoming signal to recover the information bits, which are then encoded into anther distinct codeword $\boldsymbol{c}_{2}$ by LDPC-2. Finally, $\mathrm{S}$ transmits ${ }^{c_{1}}$ and $\mathrm{R}$ transmits additional parity- check bits of $\boldsymbol{c}_{2}$ to the destination (D) to implement the Alamouti STBC scheme. Note that R sends only additional parity-check bits to $\mathrm{D}$ to retain a high-efficient coded transmission. In the destination, the incoming signals are first detected by an Alamouti combiner; then the decoder performs the joint iterative decoding algorithm with respect to the detected signals.

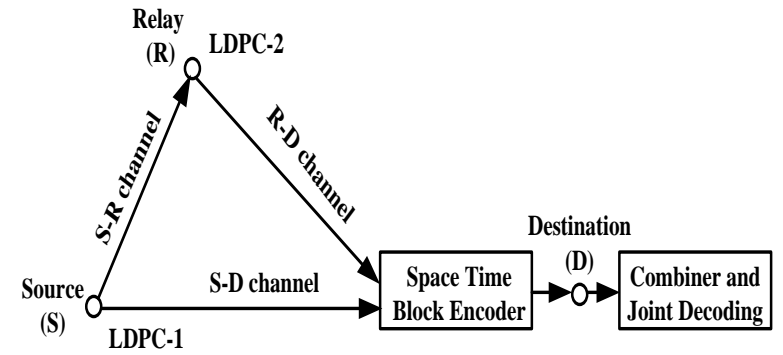

Figure 1. The proposed LDPC-coded distributed Alamouti space-time relay cooperation.

Assuming that the relay can correctly decode the signals received from the source, the ideal coded relay cooperation scheme is formulated in this paper. Using Alamouti STBC scheme in coded cooperation, the full cooperative diversity is realized by the cooperative antennas distributed in the source and relay. Moreover, the partial cooperative diversity gain contributed by channel coding arises from the decoder in destination, which estimates all the received signals, suffered from independent noises and fading in a multiple access channel, by a joint decoding means than by a separate approach. Note that the performance gain also arises from the relatively larger SNR of partial received signal from relay over a shorten distance compared with that from the remote source [12].

\section{IRREGULAR SYSTEMATIC LDPC CODES FOR DISTRIBUTED ALAMOUTI SPACE-TIME RELAY COOPERATION}

Let the sparse parity-check matrix $\boldsymbol{H}_{M \times N}$ of a binary irregular LDPC code be

$$
\boldsymbol{H}_{M \times N}=\left[\begin{array}{ll}
\tilde{\boldsymbol{H}}_{M \times(N-M)} & \boldsymbol{I}_{M \times M}
\end{array}\right]
$$


where the element of $\quad \tilde{\boldsymbol{H}}_{M \times(N-M)} \quad$ is $\quad h_{i, j} \in\{0,1\}$ $(1 \leq i \leq M, 1 \leq j \leq N-M, M<N)$ and $\boldsymbol{I}_{M \times M}$ is an identity matrix. Let the numbers of 1 's in each row and column of $\tilde{\boldsymbol{H}}_{M \times(N-M)}$ be $\tilde{d}_{c}$ and $\tilde{d}_{v}$, which are proportional to and also comparably small in contrast to $N-M$ and $\mathrm{M}$, respectively. Thus, the number of 1's in each row of $\boldsymbol{H}_{M \times N}$ is $d_{\mathrm{c}}=\tilde{d}_{c}+1$, while the numbers of 1 's in each of the first $\mathrm{N}-\mathrm{M}$ columns and of the last M columns are $d_{\mathrm{v}}=\tilde{d}_{v}$ and $d_{\mathrm{v}}=1$, respectively. Consequently, the generator matrix for the above mentioned LDPC codes is

$$
\boldsymbol{G}_{(N-M) \times N}=\left[\begin{array}{ll}
\boldsymbol{I}_{(N-M) \times(N-M)} & \tilde{\boldsymbol{H}}_{(N-M) \times M}^{\mathrm{T}}
\end{array}\right]
$$

Hence, it is obviously that a linear block code defined by the sparse parity-check matrix in (1) is an irregular systematic LDPC codes.

The task of an encoder is to generate the row vector c from the generator matrix as

$$
\boldsymbol{c}=\boldsymbol{s} \boldsymbol{G}_{(N-M) \times N}
$$

where $\mathrm{s}$ is a (N-M)-tuples message bits. Note that this linear encoding is quite suitable for the design of multiple LDPC codes applied in coded relay cooperation. In this paper, the notation $C\left(N, M, d_{c}, \tilde{d}_{v}\right)$ is used to denote the ensemble of these irregular systematic LDPC codes.

Suppose that the source and relay of a coded relay cooperation depicted in Fig.1 employ two different irregular systematic LDPC codes $C^{(1)}\left(N, M, d_{c}^{(1)}, \tilde{d}_{v}^{(1)}\right)$ and $C^{(2)}\left(2 N-M, N, d_{c}^{(2)}, \tilde{d}_{v}^{(2)}\right)$. Firstly, the encoder in the source generates the codeword $\boldsymbol{c}_{1}=\left[s_{1}, s_{2}, \cdots, s_{N-M}, p_{1}^{(1)}, p_{2}^{(1)}, \cdots, p_{M}^{(1)}\right]^{T} \quad$ including the message bits $\boldsymbol{s}=\left[s_{1}, s_{2}, \cdots, s_{N-M}\right]$ and parity-check bits $\boldsymbol{p}=\left[p_{1}^{(1)}, p_{2}^{(1)}, \cdots, p_{M}^{(1)}\right]$ by LDPC-1 defined by its paritycheck matrix as

$$
\boldsymbol{H}^{(1)}=\left[\begin{array}{ll}
\boldsymbol{A}_{M \times(N-M)} & \boldsymbol{I}_{M \times M}
\end{array}\right]
$$

Then the codeword is sent to the relay. Considering the ideal coded cooperation scenario, the decoder in the relay first correctly decodes the codeword from the received signal and then produces a distinct codeword $\boldsymbol{c}_{2}=\left[\boldsymbol{c}_{1}, p_{1}^{(2)}, p_{2}^{(2)}, \cdots, p_{N}^{(2)}\right]^{T}$ by LDPC-2 with the paritycheck matrix as

$$
\boldsymbol{H}^{(2)}=\left[\begin{array}{lll}
\boldsymbol{B}_{N \times(N-M)} & \boldsymbol{C}_{N \times M} & \boldsymbol{I}_{N \times N}
\end{array}\right]
$$

Finally, the source transmit $c_{1}$ and the relay transmit only the parity-check bits $\left[p_{1}^{(2)}, p_{2}^{(2)}, \cdots, p_{N}^{(2)}\right]$ to implement the Alamouti STBC scheme. From the view of the decoder in the destination, the whole codeword is

$$
\boldsymbol{c}=\left[\boldsymbol{c}_{1}, p_{1}^{(2)}, p_{2}^{(2)}, \cdots, p_{N}^{(2)}\right]^{T}
$$

with a block length of $2 \mathrm{~N}$, corresponding to the overall parity-check matrix as

$$
\boldsymbol{H}=\left[\begin{array}{lll}
\boldsymbol{A}_{M \times(N-M)} & \boldsymbol{I}_{M \times M} & \boldsymbol{0}_{M \times N} \\
\boldsymbol{B}_{N \times(N-M)} & \boldsymbol{C}_{N \times M} & \boldsymbol{I}_{N \times N}
\end{array}\right]
$$

Fig. 2 illustrates the joint Tanner graph corresponding to the overall parity-check matrix defined by (7). In this figure, the 1st and 2nd layer in the joint Tanner graph are associated with the check nodes $c_{i}^{(1)}(1 \leq i \leq M)$ and $c_{i}^{(2)}(1 \leq i \leq N)$ related to the LDPC codes $C^{(1)}\left(N, M, d_{c}^{(1)}, \tilde{d}_{v}^{(1)}\right)$ and $C^{(2)}\left(2 N-M, N, d_{c}^{(2)}, \tilde{d}_{v}^{(2)}\right)$, respectively, where each check node in the 1 st and 2 nd layer involves with ${ }^{d_{c}}$ and $d_{c}^{(1)}$ variable nodes, respectively. The third layer consists of two distinct related sublayers of total $2 N$ variable nodes. In the 1 st sublayer, each of the $N-M$ variable nodes $v_{i}^{(1)}$ $(1 \leq i \leq N-M)$ participates in $\tilde{d}_{v}+\tilde{d}_{v}^{(1)}$ check equations, while each of $\mathrm{M}$ variable nodes $v_{i}^{(1)}(N-M+1 \leq i \leq N)$ participates in only one check equation. In the second sublayer, each of $N$ variable nodes $v_{i}^{(2)}(N+1 \leq i \leq 2 N)$ also only attends one check equation.

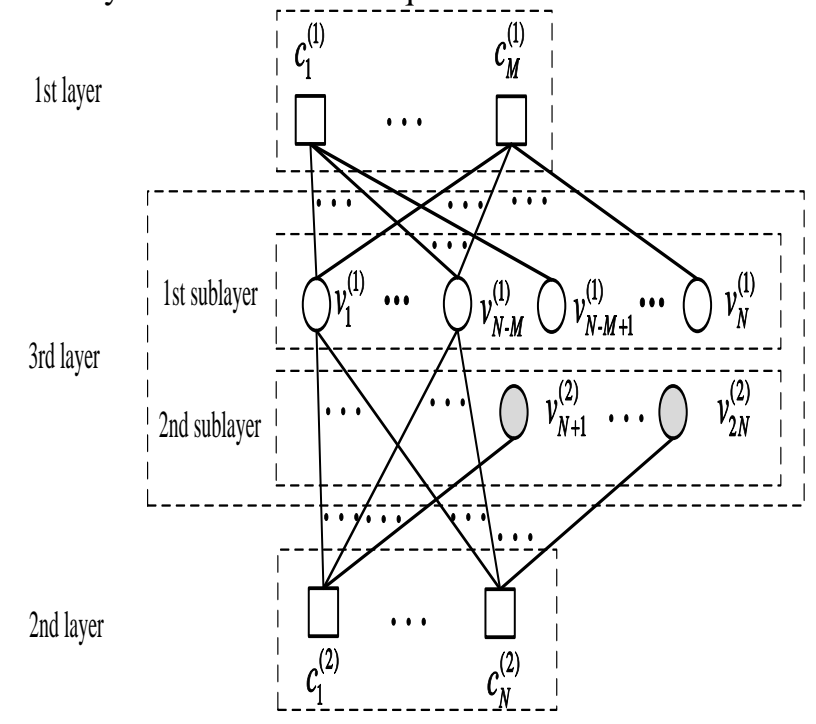

Figure 2. The joint Tanner graph for double irregular systematic LDPC codes adopted by a distributed Alamouti space-time coded relay cooperation. 


\section{ALAMOUTI COMBINER AND JOINT ITERATIVE DECODING IN THE DESTINATION}

\section{A. The Combining Scheme for Alamouti STBC}

In the proposed coded relay cooperation scheme shown in Fig. 1, the source and relay send their signals to the destination based on Alamouti STBC to achieve full cooperative diversity. At time $\mathrm{k}$, two symbols denoted by $d_{S, k}$ and $d_{R, k}$ are simultaneously transmitted from the two antennas distributing in the source and relay, respective. During the next symbol period signal $\left(-d_{R, k}^{*}\right)$ is transmitted from the source, while signal $d_{S, k}^{*}$ is transmitted from the relay where * is the complex conjugation operation. Hence the received signal at time $\mathrm{k}$ at the destination can be expressed as

$$
r_{D, k}=h_{S D, k} d_{S, k}+\sqrt{g_{R D}} h_{R D, k} d_{R, k}+n_{k}
$$

where $h_{S D, k}$ and $h_{R D, k}$ are zero-mean unit-variance complex Gaussian random variables (RV) for the Rayleigh fading channel associated with the source and destination (S-D) and the relay to the destination (R-D), respectively, $n_{k}$ is a complex Gaussian noise with zero-mean and variance- $N_{0}$, $g_{R D}$ is the power-gain [12].

Assuming that the fading is constant across two consecutive symbols, consequently, the received signal at time $k+T$ at the destination can be formulated as

$$
r_{D, k+T}=-h_{S D, k} d_{R, k}^{*}+\sqrt{g_{R D}} h_{R D, k} d_{S, k}^{*}+n_{k+T}
$$

where $\mathrm{T}$ is the symbol duration and $n_{k+T}$ is a complex Gaussian noise with zero-mean and variance- $N_{0}$.

In the destination, the Alamouti combiner builds the following two combined signals which will be sent to the joint BP decoder:

$$
\begin{gathered}
y_{S, k}=h_{S D, k}^{*} r_{D, k}+\sqrt{g_{R D}} h_{R D, k} r_{D, k+T}^{*} \\
y_{R, k}=\sqrt{g_{R D}} h_{R D, k}^{*} r_{D, k}-h_{S D, k} r_{D, k+T}^{*}
\end{gathered}
$$

Substituting (8) and (9) into (10) and (11) we get

$$
\begin{gathered}
y_{S, k}=\left(\left|h_{S D, k}\right|^{2}+g_{R D}\left|h_{R D, k}\right|^{2}\right) d_{S, k}+\tilde{n}_{k} \\
y_{R, k}=\left(\left|h_{S D, k}\right|^{2}+g_{R D}\left|h_{R D, k}\right|^{2}\right) d_{R, k}+\tilde{n}_{k+T}
\end{gathered}
$$

where we introduce $\tilde{n}_{k}=h_{S D, k}^{*} n_{k}+\sqrt{g_{R D}} h_{R D, k} n_{k+T}^{*}$ and $\tilde{n}_{k+T}=\sqrt{g_{R D}} h_{R D, k}^{*} n_{k}-h_{S D, k} n_{k+T}^{*}$ while $\tilde{n}_{k}$ and $\tilde{n}_{k+T}$ are complex Gaussian random variable with zero-mean and variance $\sigma^{2}$ calculated by

$$
\sigma^{2}=\left(\left|h_{S D, k}\right|^{2}+g_{R D, k}\left|h_{R D, k}\right|^{2}\right) N_{0}
$$

Note that the two combined sequences $\left(y_{S, k}\right)$ and $\left(y_{R, k}\right)$ will be used as the inputs in the subsequent BP-based LDPC iterative decoder.

\section{B. Joint BP-Based Iterative Decoding Based on Multi- \\ Layer Tanner Graph for Double Irregular Systematic LDPC Codes}

In order to be well presented, the aforementioned two detected sequences $\left(y_{S, k}\right)$ and $\left(y_{R, k}\right)$ are re-arranged as the following one complex sequence

$$
\boldsymbol{R}=\left(y_{1}, \ldots, y_{N}, y_{N+1}, \ldots, y_{2 N}\right)
$$

by ignoring the time index $\mathrm{k}$ without causing any confusion, where the first and last $\mathrm{N}$ symbols from are corresponding to LDPC $\quad$ codes $\quad C^{(1)}\left(N, M, d_{c}^{(1)}, \tilde{d}_{v}^{(1)}\right)$ and $C^{(2)}\left(2 N-M, N, d_{c}^{(2)}, \tilde{d}_{v}^{(2)}\right)$ used by the source and relay, respectively. Consequently, by (12) and (13) $y_{n}$ $(n=1, \cdots, 2 N)$ is expressed as

$$
y_{n}=\left\{\begin{array}{lr}
\left(\left|h_{n}\right|^{2}+g_{n+N}\left|h_{n+N}\right|^{2}\right) d_{n}+\tilde{n}_{n} & 1 \leq n \leq N \\
\left(\left|h_{n-N}\right|^{2}+g_{n}\left|h_{n}\right|^{2}\right) d_{n}+\tilde{n}_{n} & N<n \leq 2 N
\end{array}\right.
$$

for different categories of $\mathrm{n}$, where ${ }^{d_{n}}$ takes values of +1 or -1 with equal likelihood for the codeword bit $c_{n}=0$ or 1 generated by irregular LDPC code $C^{(1)}\left(N, M, d_{c}^{(1)}, \tilde{d}_{v}^{(1)}\right)$ or $C^{(2)}\left(2 N-M, N, d_{c}^{(2)}, \tilde{d}_{v}^{(2)}\right)$ used by the source and relay, respectively, $h_{n}$ and $\tilde{n}_{n}$ are independent Rayleigh fading and Gaussian RVs with variance ${ }^{2}$ given in (14), respectively, while $g_{n}$ is the power gain.

In this research $\boldsymbol{R}$ is reduced to a real sequence as

$$
\boldsymbol{R}=\left(\tilde{r}_{n}\right)=\left(\operatorname{Re}\left(y_{n}\right)\right)(1 \leq n \leq 2 N)
$$

when binary antipodal modulation is employed. Initially, the decoder only obtains the combined signals from the STBC combiner and has no prior information from the parity 
checks. Thus with the known CSI, the joint iterative decoding [8] based on the described joint Tanner graph will be done after the decoder initialized by $f_{n}$ expressed as

$$
\begin{aligned}
& f_{n}=\operatorname{Pr}\left(c_{n}=0 \mid\left(\left.\tilde{r}_{l}\right|_{l=1} ^{2 N}\right)=\operatorname{Pr}\left(c_{n}=0 \mid \tilde{r}_{n}\right)\right. \\
& = \begin{cases}\frac{1}{1+\exp \left\{\left[\left(-4\left(\left|h_{n}\right|^{2}+g_{n+N}\left|h_{n+N}\right|^{2}\right) \tilde{r}_{n}\right] / \sigma^{2}\right\}\right.} & 1 \leq n \leq N \\
\frac{1}{1+\exp \left\{\left[\left(-4\left|h_{n-N}\right|^{2}+g_{n}\left|h_{n}\right|^{2}\right) \tilde{r}_{n}\right] / \sigma^{2}\right\}} & N+1 \leq n \leq 2 N\end{cases}
\end{aligned}
$$

for different categories of $\mathrm{n}$.

\section{SIMULATION RESULTS}

We investigate the BER performance of proposed LDPCcoded distributed Alamouti space-time relay cooperation over a Rayleigh fading channel. The simulation results are shown in Figs. 3 4, respectively, where we assume that the power gain is $1.0 \mathrm{~dB}$ (i.e. $10 \log g_{R D}=1.0 \mathrm{~dB}$ ) and BPSK modulation is adopted.

\section{A. LDPC-Coded Distributed Alamouti Space-Time Relay Cooperation with Various Decoding Iterations}

Fig. 3 gives BER results of the proposed LDPC-coded distributed Alamouti space-time relay cooperation with 1, 3 and 10 decoding iterations by the proposed concatenation of STBC combiner and joint BP-based decoder, where two different irregular systematic LDPC codes $C^{(1)}(720,240,4,2)$ and $C^{(2)}(1200,720,3,3)$ are employed for the source and relay, respectively. Simulation results show that the BER can be significantly reduced by using the joint BP-based iterative decoder with increasing decoding iterations. For example, at a BER of $10^{-3}$ approximate 1.2 $\mathrm{dB}$ gain can be achieved for the 3rd iteration over the 1st iteration, while the gain is about $0.7 \mathrm{~dB}$ for the 10 th iteration as compared to the 3rd iteration at the same BER.

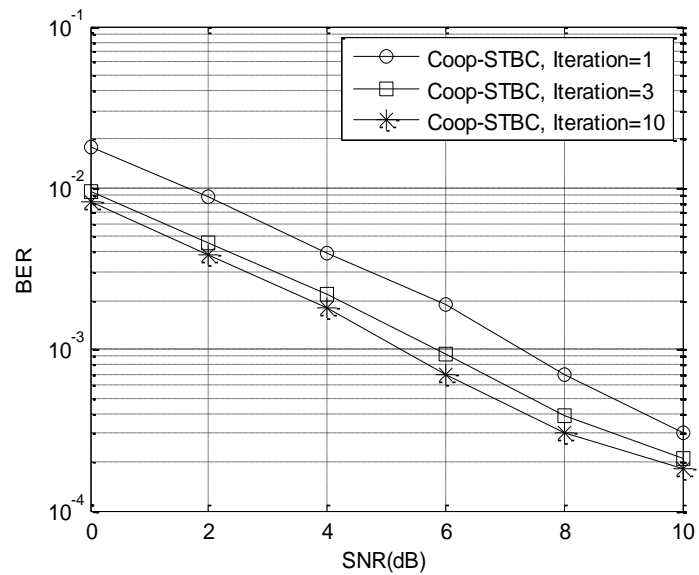

Figure 3. BER of LDPC-coded distributed Alamouti space-time relay cooperation scheme with various decoding iterations in the destination over a Rayleigh fading channel.

\section{B. LDPC-Coded Relay Cooperation with and without Alamouti STBC Processing}

Fig. 4 compares the BER of LDPC-coded relay cooperation with and without [8, Fig.11] Alamouti STBC by using the same LDPC codes as in Fig. 3 by ten decoding iterations. It is clear that our approach with Alamouti STBC processing provides a significant performance gains over the other approach under the same conditions in all ranges of SNRs.

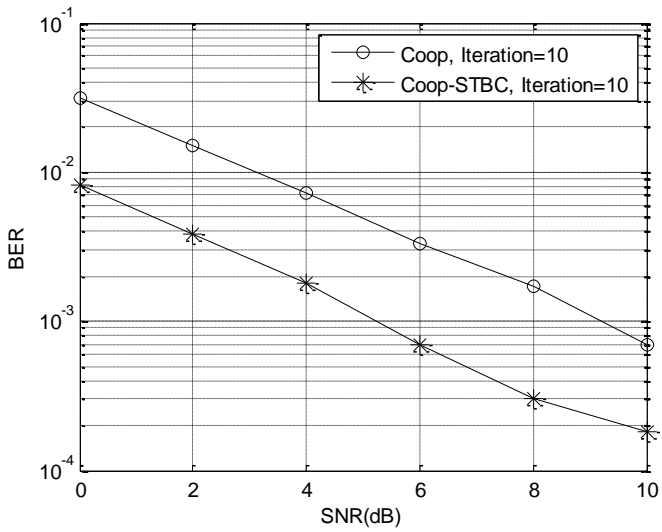

Figure 4. BER of LDPC-coded cooperation with and without Alamouti STBC processing by ten decoding iterations over a Rayleigh fading channel.

\section{CONCLUSION}

We propose an efficient LDPC-coded distributed Alamouti space-time relay cooperation scheme over a Rayleigh fading channel, which can combine the advantages of diversity gain and coding gain into one unit by STBC combiner and the introduced BP-based joint iterative decoding in the destination. Theoretical analysis and simulation results demonstrate that the proposed coded cooperation significantly outperforms over the conventional coded cooperations in lower BER and simple encoding.

\section{ACKNOWLEDGMENT}

This work is supported by the School Scientific Research Fund of Nanjing Institute of Technology (NO. YKJ201417 and YKJ201419).

\section{REFERENCES}

[1] A. Sendonaris, E. Erkip, and B. Aazhang, "User cooperation diversity-Part I: System description," IEEE Trans. Commun., vol. 51, no. 11, pp.1927-1938, Nov. 2003.

[2] Laneman J, Wornell G and Tse D, "An efficient protocol for realizing cooperative diversity in wireless networks," Proceedings IEEE International Symposium on Information Theory 2001, Washing DC, U.S.A., June 2001, pp.294-297.

[3] Cover T and Gamal A, "Capacity theorems for the relay channel," IEEE Trans. on Inf. Theory, 1979, vol. 25, no.5, pp.572-584.

[4] T. E. Hunter and A. Nosratinia, "Diversity through coded cooperation," IEEE Trans. Wireless Commun., vol. 5, no. 2, Feb. 2006, pp. 283-289.

[5] V. Savin "Split-extended LDPC codes for coded cooperation," Information Theory and its Applications (ISITA), vol. 5, no. 2, Feb. 2010, pp. 151-156. 
[6] Z.-Q. Chen, S. Ouyang and H. Xiao, "The design of efficientlyencodable LDPC codes for coded cooperation in relay channel," Communications and Mobile Computing, vol. 53, no. 10, Oct. 2010, pp. 221-223.

[7] Ishibashi, Koji; Ishii, Koji; Ochiai and Hideki; "Dynamic coded cooperation using multiple turbo codes in wireless relay networks," IEEE Journal on Selected Topics in Signal Processing, vol. 5, no.1, pp.197-207, 2011.

[8] Lei Tang, Fengfan Yang, Shunwai Zhang and Hongjun Xu, "Joint iterative decoding for LDPC-coded multi-relay cooperation with receive multi-antenna in the destination," IET Communications, 2013, vol.7, no.1, pp.1-12.

[9] A. A. Nasir; H. Mehrpouyan; S. Durrani; S. D. Blostein; R. A. Kennedy and B. Ottersten, "Transceiver design for distributed STBC based AF cooperative networks in the presence of timing and frequency offsets," IEEE Transactions on Signal Processing, 2013, vol. 61, no.12, pp. 3143-3158.

[10] C. Abou-Rjeily, "Distributed space-time codes for full-duplex IRUWB amplify-and-forward cooperation," IEEE Transactions on Wireless Communications, 2015, vol.14, no.4, pp. 2144-2155.

[11] N. Varshney; A. V. Krishna; A. K. Jagannatham, "Selective DF protocol for MIMO STBC based single/multiple relay cooperative communication: end-to-end performance and optimal power allocation," IEEE Transactions on Communications, 2015, vol. 63, no. 70 , pp. 2458-2474.

[12] L. Wang, L-K Kong, X.-N. Soon and L. Hanzo, "A near-capacity differentially encoded non-coherent adaptive multiple-symboldetection aided three-stage coded scheme," Vehicular Technology Conference' 71 , 2010, p.1-5, 16-19. 